\title{
ALTERAÇÃO DA TEMPERATURA OCULAR EM RESPOSTA AO TREINAMENTO DE CORRIDA EM CAVALOS PURO-SANGUE-INGLÊS NO JOCKEY CLUB
}

\section{(Eye temperature change in response to race training in thoroughbred horses at the Jockey Club)}

\author{
Jéssica Carvalho Seabra ${ }^{1}$, João Ricardo Dittrich ${ }^{1}$, Marcos Martinez do Vale ${ }^{1}$, Jéssica do Rocio \\ Janiszewski ${ }^{1}$, Rafaella Silva de Hollanda ${ }^{2}$ \\ 'Universidade Federal do Praná, ${ }^{2}$ Universidade Positivo
}

Corresponding author: jessicacseabra@yahoo.com.br

\begin{abstract}
RESUMO: Durante uma situação estressante, a termografia infravermelha pode detectar as alterações do fluxo sanguíneo causadas pela liberação de catecolaminas e cortisol. O objetivo deste estudo foi avaliar as alterações da temperatura ocular associadas ao estresse fisiológico induzido por uma sessão de treinamento de corrida com cavalos Puro-sangue-inglês. Imagens térmicas do olho esquerdo de 13 cavalos puro-sangue-inglês foram capturadas antes e depois do exercício durante o último dia de treinamento antes da primeira corrida oficial dos animais e a temperatura máxima do globo ocular foi analisada. A frequência respiratória dos animais foi mensurada antes e após o exercício, sendo registradas também a temperatura ambiente e a umidade relativa do ar no estábulo e na pista de corrida. $O$ experimento foi realizado em delineamento inteiramente casualizado com 13 repetições. As mudanças na temperatura máxima ocular foram analisadas com ANOVA e a correlação entre a temperatura máxima ocular, a frequência respiratória e o índice de conforto térmico (IC) foi investigada. Houve aumento significativo $(\mathrm{P}<0,01)$ da temperatura ocular após a sessão de treinamento. Foi encontrada uma correlação linear positiva entre a frequência respiratória e as temperaturas máximas oculares, entretanto não houve correlação entre a temperatura ocular e o índice de conforto térmico ou entre a frequência respiratória e o índice de conforto térmico. A termografia infravermelha é capaz de medir efetivamente a mudança de temperatura do olho, que pode estar relacionada aos níveis de estresse em cavalos puro-sangue durante o treinamento de corrida.
\end{abstract}

Palavras-chave: bem-estar; cavalos atletas; equinos; exercício; termografia infravermelha.

ABSTRACT: During a stressful situation, the infrared thermography can detect the alterations of the blood flow caused by the release of catecholamines and cortisol. The goal of this study was to evaluate the eye temperature change associated with physiological stress in response to a session of race training in thoroughbred horses. Thermal images of the left eye of 13 thoroughbred horses were captured before and after the last training day before their first official race and maximum eye temperature was analyzed. The respiratory rate before and after exercise was also measured and the ambient temperature and the relative humidity of the air at the stable and at the track were recorded. The experiment was performed under a complete randomized design with 13 replications. The analysis of changes in maximal eye temperature was done with an ANOVA procedure and a correlation between the maximal eye temperature, respiratory rate and Comfort Index was investigated. There was a significant increase $(P<0.01)$ in the eye temperature 
measurements taken after the training session. There was a positive linear correlation between the respiratory rate and the maximal eye temperatures, but correlations between the Comfort Index and maximum eye temperature or between the respiratory rate and the Comfort Index were not found. The infrared thermography is able to effectively measure the eye temperature change, which may be related to stress levels in thoroughbred horses during race training.

Keywords: athletic horses; equine; exercise; infrared thermography; welfare. 


\section{INTRODUÇÃO}

Durante o exercício, o cavalo precisa aumentar a sua taxa metabólica para transportar oxigênio, água, eletrólitos, nutrientes e hormônios até os músculos em contração, além de remover da corrente sanguínea dióxido de carbono e outros metabólitos. A conversão da energia química (ou seja, substratos armazenados) para energia mecânica (por exemplo, contração muscular) é ineficiente, com aproximadamente $75-80 \%$ da energia química total sendo liberada em forma de calor em vez de trabalho físico (Brody, 1945). Com o início do exercício físico, há aumento da taxa de produção de calor metabólico, acompanhada por elevação da temperatura muscular e temperatura corporal interna que, se não efetivamente dissipada, resulta em hipertermia. Essas alterações sistêmicas causadas pelo exercício desencadeiam respostas integradas em todo o corpo, requerendo mediações neurais e endócrinas que visam manter o ambiente interno dentro de limites relativamente estreitos, provendo condições ótimas para a função celular (McKeever, 2002). A sobrecarga desses processos fisiológicos leva ao distúrbio da homeostase corporal, fazendo com que o exercício seja considerado um estímulo estressante. Embora o termo "estresse" tenha uma conotação negativa, alguns autores sugerem que, sob certas circunstâncias, as exposições ao estresse podem ter 0 potencial de melhorar o desempenho e a resiliência de um organismo, produzindo resultados finais positivos e sendo chamado de eustresse. $O$ estresse negativo ocorre quando um indivíduo é confrontado com um estressor agudo ou crônico, que ultrapassa suas capacidades de adaptação, sendo chamado de distresse (Moberg, 2000; Peeters, 2013).
Segundos após perceber um estressor, dois sistemas são ativados: o sistema nervoso simpático (SNS), seguido pela estimulação do eixo hipotálamo-hipófise-adrenal (HPA), preparando o cavalo para uma resposta imediata que é denominada como reação de "luta ou fuga". O cortisol é produzido pelo córtex da adrenal, enquanto a norepinefrina (noradrenalina) é sintetizada pela poção medular da adrenal. No entanto, a noradrenalina (adrenalina) pode ser secretada pelas terminações nervosas simpáticas, além de ser produzida pela medula da glândula adrenal. $\mathrm{O}$ aumento da secreção de catecolaminas e cortisol é responsável por preparar o cavalo para a atividade física intensa envolvida em uma reação de luta ou fuga. Esses hormônios induzem à dilatação das pupilas, aumentam a frequência e a força da contração muscular cardíaca, promovem a constrição de alguns leitos vasculares e o aumento da pressão sanguínea (Porges, 1995). O sangue é desviado de áreas que não são consideradas importantes para a resposta de luta e fuga, incluindo o trato gastrointestinal e o sistema reprodutivo, sendo redirecionado aos órgãos sensoriais como o músculo esquelético, pulmões, coração e encéfalo. A vasoconstrição da pele também pode ser um mecanismo de proteção que visa reduzir a perda de sangue caso ocorram ferimentos (Blessing, 2003).

O monitoramento de respostas fisiológicas, como a atividade do eixo hipotálamo-hipófise-adrenal (HPA) e mudanças nas concentrações plasmáticas de cortisol são frequentemente usadas para estudar as reações ao estresse em animais de fazenda, (Broom e Johnson, 1993). Entretanto, alguns indicadores fisiológicos são de difícil mensuração, como os níveis hormonais e de atividade imunológica, pois estes métodos requerem coletas de sangue, 
processo este que pode causar estresse e alterar os resultados. Além disso, a maioria dessas técnicas requerem instrumentos altamente especializados e um longo período de tempo dedicado às análises, sendo às vezes necessárias condições específicas para realizar a coleta do material no animal (Stewart et al., 2005). Consequentemente, faz-se imprescindível o desenvolvimento de uma ferramenta não invasiva para mensurar os níveis de estresse em cavalos durante competições equestres.

Estudos anteriores apontam que a termografia infravermelha tem um bom potencial como uma ferramenta não invasiva para a mensuração dos níveis de estresse em cavalos durante competições (Valera et al., 2012.; McGreevy, Warren-Smith and Guisard, 2012; Bartolomé et al., 2013). A secreção de hormônios durante uma situação estressante altera o fluxo sanguíneo e, consequentemente, modifica a produção e a perda de calor da superfície corporal do animal (Schaefer et al., 2002), sendo possível detectar essas mudanças de temperatura utilizando a câmera termográfica. Especificamente, a temperatura do olho e pequenas áreas ao redor da borda posterior da pálpebra e da carúncula lacrimal, que são áreas ricas em capilares inervados pelo sistema simpático e por isso respondem rapidamente a mudanças no fluxo sanguíneo. A termografia infravermelha já foi utilizada por pesquisadores para medir mudanças na temperatura ocular de cavalos. Cook et al. (2001) relataram o aumento da temperatura ocular, além de encontrarem uma correlação positiva entre a temperatura máxima ocular e os níveis de cortisol salivar e plasmático, sugerindo que mudanças na temperatura ocular podem ser impulsionadas pela ativação do eixo hipotalâmico-pituitário-adrenal.
Apesar de a temperatura máxima ocular não ser capaz de definir a natureza do estímulo estressante (euestresse ou distresse) quando utilizada como parâmetro único, a variação de temperatura poderia ser uma consequência das mudanças hormonais e refletir a intensidade da ativação dos sistemas alostáticos que visam manter a estabilidade do meio interno. Portanto, o objetivo deste estudo foi avaliar a alteração da temperatura máxima ocular associada ao estresse fisiológico em resposta a uma sessão de treinamento de corrida em cavalos puro-sangue-inglês no Jockey Club.

\section{MATERIAL E MÉTODOS}

\section{Animais e local do estudo}

Todos os procedimentos realizados no experimento foram aprovados pelo Comitê de Ética no uso de animais da Universidade Federal do Paraná (UFPR, protocolo número 033/2017). Este experimento utilizou oito éguas e cinco garanhões da raça Puro-Sangue-Inglês alojados em baias individuais no Jockey Club do Paraná, em Curitiba, Brasil.

Os equinos tinham cerca de dois anos e pesavam $460 \pm 45 \mathrm{Kg}$. Eles compartilhavam o mesmo manejo e a rotina de alimentação era composta de três refeições por dia de feno de alfafa e uma mistura de ração concentrada e aveia.

\section{Treinamento de corrida}

Após serem trazidos para o Jockey Club, os cavalos foram domados, sendo em seguida introduzidos ao protocolo de treinamento de corrida. Durante todo o treinamento, os cavalos foram exercitados cinco dias por semana durante um tempo médio de 20 minutos e durante um período total de cinco 
meses. As imagens térmicas do olho esquerdo foram capturadas antes e depois do último dia de treinamento, antes da primeira corrida oficial dos cavalos. A sessão de treinamento no dia experimental durou 20 minutos e foi caracterizada por 900 metros de trote (cerca de $3,4 \mathrm{~m} / \mathrm{s}$ ) e 1000 metros de galope rápido (cerca de $11 \mathrm{~m} / \mathrm{s}$ ).

\section{Coleta de Dados}

A temperatura e a umidade relativa do ar foram registradas em intervalos de cinco minutos por dois dataloggers, um colocado no estábulo e outro colocado na pista de corrida. Um histograma foi produzido mostrando a temperatura e umidade ao longo do tempo e esses dados foram utilizados para avaliar as condições ambientais, calculando o Índice de Conforto térmico para cavalos (IC) (Jones, 2009), como mostrado na equação: $\mathrm{Cl}=\mathrm{T}+\mathrm{RH}$

Onde: $\mathrm{T}_{\mathrm{ar}}$, temperatura de bulbo seco em $\left({ }^{\circ} \mathrm{F}\right)$;

$\mathrm{RH}$, umidade relativa do ar (\%)

O Índice de Conforto Térmico (IC) foi utilizado para monitorar o potencial efeito do microclima como fator contribuinte para a mudança térmica. As medidas da temperatura e da umidade relativa no momento específico em que cada imagem térmica foi coletada também foram inseridas no software para calibrar a análise das imagens. Além disso, a frequência respiratória dos animais também foi medida antes e após 0 exercício utilizando um estetoscópio.

Uma câmera térmica infravermelha portátil (Fluke Ti-25) foi usada para coletar imagens oculares em dois momentos distintos: antes da sessão de treinamento e logo após. Antes de serem levados para a pista de corrida e depois de voltarem para os estábulos, os cavalos foram conduzidos individualmente para dentro do boxe de ducha, onde as imagens foram tiradas.
O olho esquerdo de todos os cavalos foi escaneado a partir de um ângulo de $90^{\circ}$ e a uma distância de $1 \mathrm{~m}$, sendo utilizado o índice de emissividade de 0,95 . Diversas imagens foram registradas por animal, sendo selecionada a imagem que forneceu as melhores condições de operação para análise. O software SmartView (Fluke Corporation, Everett, WA, EUA) foi utilizado para analisar as imagens da câmera termográfica. A temperatura máxima ocular $\left({ }^{\circ} \mathrm{C}\right)$ utilizada para as análises foi mensurada dentro de uma área oval traçada ao redor do olho do animal, incluindo o globo ocular e cerca de $1 \mathrm{~cm}$ ao redor das pálpebras (Valera et al., 2012).

\section{Delineamento Experimental e Análises Estatísticas}

O experimento foi conduzido em delineamento inteiramente casualizado com 13 repetições. $A$ análise das alterações da temperatura máxima ocular foi realizada com ANOVA e o teste de Bartlett foi utilizado para verificar a homogeneidade das variâncias. O nível de significância aceito foi $P<0,05$. A relação entre a temperatura máxima ocular, o Índice de Conforto Térmico (IC) e a frequência respiratória foi investigada usando a correlação linear simples. O programa estatístico Assistat, 2016 (The Assistat Software Version 7.7) foi utilizado para a realização de todas as análises estatísticas.

\section{RESULTADOS}

Houve diferença significativa $(P<0,01)$ entre as medidas de temperatura ocular realizadas antes e após a sessão de treinamento (Tabela 1). As temperaturas máximas oculares foram significativamente maiores após o treinamento de corrida. As temperaturas máximas oculares de todos os cavalos 
antes do exercício variaram de $33,56^{\circ} \mathrm{C}$ a $35,67^{\circ} \mathrm{C}$, com valor médio de $34,59^{\circ} \mathrm{C}$, enquanto a temperatura máxima ocular após o exercício variou de $34,94^{\circ} \mathrm{C}$ a $37,56^{\circ} \mathrm{C}$, com valor médio de $36,22^{\circ} \mathrm{C}$. A temperatura ambiente e a umidade relativa do ar registradas no estábulo e na pista de corrida ao longo da duração do experimento podem ser vistas na
Tabela 2. Houve uma correlação linear positiva entre a temperatura máxima ocular e a frequência respiratória, mas correlações entre o Índice de Conforto Térmico e a temperatura máxima ocular ou entre a frequência respiratória e o Índice de Conforto Térmico não foram encontradas (Tabela 3).

Tabela 1 - Média das temperaturas máximas oculares $\left({ }^{\circ} \mathrm{C}\right)$, frequência respiratória e Índice de Conforto Térmico dos cavalos antes e após o exercício.

\begin{tabular}{cccc|ccc}
\hline & \multicolumn{3}{c|}{ Antes do Exercício } & \multicolumn{3}{c}{ Após o exercício } \\
\cline { 2 - 7 } & $\begin{array}{c}\text { Valor } \\
\text { Mínimo }\end{array}$ & \multicolumn{1}{c}{ Média } & $\begin{array}{c}\text { Valor } \\
\text { Máximo }\end{array}$ & $\begin{array}{c}\text { Valor } \\
\text { Mínimo }\end{array}$ & Média & $\begin{array}{c}\text { Valor } \\
\text { Máximo }\end{array}$ \\
\hline $\begin{array}{c}\text { Temperatura } \\
\text { Ocular Máxima } \\
\left({ }^{\circ} \mathrm{C}\right)\end{array}$ & 33,56 & $34,59 \pm 0,81$ & 35,67 & 34,94 & $36,22 \pm 0,90^{* * 2}$ & 37,56 \\
\hline $\begin{array}{c}\text { Frequência } \\
\text { Respiratória } \\
(\text { mov/min) }\end{array}$ & 12,00 & $19,69 \pm 6,42$ & 32,00 & 24,00 & $55,08 \pm 19,61$ & 92,00 \\
\hline $\begin{array}{c}\text { Índice de } \\
\text { Conforto Térmico }\end{array}$ & 151,00 & $154,15 \pm 3,42$ & 159,50 & 152,40 & $155,35 \pm 2,35$ & 159,50 \\
\hline 1 - mov/min= movimentos por minuto, 2- **significativo ao nível de 1\% de probabilidade $(p<0,01)$ & &
\end{tabular}

Tabela 2 - Temperatura ambiente média e umidade relativa do ar (RH\%) no estábulo e na pista de corrida.

\begin{tabular}{cccc}
\hline & Valor Mínimo & Média & $\begin{array}{c}\text { Valor } \\
\text { Máximo }\end{array}$ \\
\hline Temperatura Ambiente Estábulo $\left({ }^{\circ} \mathrm{C}\right)$ & 20,70 & $24,45 \pm 2,46$ & 30,00 \\
RH Estábulo $(\%)$ & 68,80 & $78,08 \pm 7,20$ & 89,80 \\
Temperatura ambiente na Pista $\left({ }^{\circ} \mathrm{C}\right)$ & 18,60 & $21,63 \pm 2,46$ & 26,50 \\
RH Pista $(\%)$ & 69,20 & $87,39 \pm 8,51$ & 97,20 \\
\hline
\end{tabular}

Tabela 3 - Coeficiente de correlação calculado entre máxima temperatura ocular e frequência respiratória, máxima temperatura ocular e índice de conforto térmico e entre a frequência respiratória e o índice de conforto térmico.

Temperatura Máxima Ocular X Frequência Respiratória

Temperatura Máxima Ocular X Índice de Conforto Térmico

Frequência Respiratória $X$ Índice de conforto Térmico

$0,5909 * * 1$
$0,0979 \mathrm{~ns}^{2}$
$-0,0460 \mathrm{~ns}^{2}$

1 - ** significante ao nível de $1 \%$ de probabilidade $(p<0.01), 2-n s=$ não significativo

\section{DISCUSSÃO}

Mudanças na temperatura ocular relacionadas a estímulos estressantes (que causam distúrbio da homeostase corporal), como observado neste experimento, foram relatadas em vários estudos anteriores com bovinos (Stewart et al., 2008) e equinos (Yarnell, 2011; McGreevy, Warren-Smith e
Guisard, 2012; Valera et al., 2012; Bartolomé et al., 2013;). Yarnell (2012) investigou a resposta fisiológica dos cavalos ao procedimento de tosa, que é comumente empregado em animais de esporte. Ela observou aumento significativo da temperatura ocular ao longo da duração do procedimento de tosa em todos os cavalos, havendo uma resposta mais significativa durante os 
cinco minutos após o início da tosa, sugerindo que $o$ aumento da temperatura ocular possa ter sido gerado pelo sistema nervoso simpático devido à resposta rápida a partir do início do estímulo estressor. Este estudo também encontrou uma correlação positiva entre o aumento da temperatura ocular e o aumento dos níveis de cortisol salivar durante o procedimento de tosa, indicando que o procedimento foi aversivo para os cavalos e, portanto, - aumento na temperatura ocular poderia ser uma consequência a um estímulo estressante. Resultados semelhantes também foram encontrados em um estudo que avaliou a resposta ao estresse de equinos durante competições de saltos. Valera et al. (2012) também encontraram correlações positivas entre a temperatura máxima ocular e o aumento dos níveis de cortisol salivar, concluindo que ambas as técnicas são capazes de medir uma resposta fisiológica a estímulos estressores externos.

Experimentos científicos em que a termografia infravermelha foi utilizada para medir a mudança de temperatura ocular como resposta a situações estressantes em equinos têm encontrado resultados relativamente constantes. Bartolomé et al. (2013) observaram aumento da temperatura ocular em cavalos antes e após uma competição de saltos, registrando variações entre as temperaturas de $36^{\circ} \mathrm{C}$ a $38^{\circ} \mathrm{C}$, enquanto Yarnell (2011) encontrou uma variação de $28,3^{\circ} \mathrm{C}$ a $37,5^{\circ} \mathrm{C}$ na temperatura ocular de equinos submetidos ao procedimento de tosa e Hall et al. (2011) relataram uma variação de $32,34 \pm 2,61^{\circ} \mathrm{C}$ após uma sessão de exercícios na guia. Estes resultados são consistentes com o que foi encontrado durante o estudo atual, em que a temperatura máxima ocular variou de $33,56^{\circ} \mathrm{C}$ a $37,56^{\circ} \mathrm{C}$ antes e depois da sessão de treinamento de corrida.
Alguns autores sugerem que as condições ambientais podem ser um fator potencialmente limitante no uso da termografia infravermelha. Church et al. (2014) observaram que condições ambientais como distância, velocidade do vento e radiação solar podem influenciar os valores de temperatura ocular mensurados com a câmera termográfica. No entanto, os mesmos autores sugerem que esses efeitos poderiam ser minimizados através do uso de abrigos ou telas. Embora as imagens do presente estudo tenham sido coletadas durante todo o período da manhã, quando a temperatura ambiente variou de 20,70 a $30,00^{\circ} \mathrm{C}$, não foi encontrada uma correlação entre o Índice de Conforto Térmico e as temperaturas máximas oculares, indicando que as condições ambientais no local onde as imagens foram coletadas estavam estáveis e não afetaram a mensuração da temperatura máxima ocular.

Segundo Jones (2009), se o valor calculado para o Índice de Conforto Térmico $(\mathrm{Cl})$ for menor que 130 , os equinos estão dentro da sua zona termoneutra, significando que o sistema termorregulatório desses animais não será ativado. No entanto, o valor calculado para o Índice de Conforto Térmico (IC) durante a coleta das imagens antes e após a sessão de exercício foi de 154,15 $\pm 3,42$ e $155,35 \pm 2,35$, respectivamente, caracterizando uma situação de desconforto térmico pelo calor. Entretanto, estudos realizados anteriormente com o objetivo de estimar a zona termoneutra para equinos apresentaram resultados inconsistentes (McBride et al., 1984; Clarke., 1987; Morgan et al., 1997). Essas variações demonstram que a zona termoneutra dos cavalos é dependente de vários fatores, como raça, região, estação do ano e adaptação. Os cavalos utilizados neste experimento nasceram e foram 
criados em território brasileiro, sendo aclimatados a condições quentes e úmidas. Nesse tipo de clima, os equinos não aclimatados aumentam a proporção do calor que é dissipado pelo trato respiratório devido à elevação da frequência respiratória que pode representar $25 \%$ ou mais da perda total de calor nessas condições. Embora o Índice de Conforto Térmico tenha indicado estresse por calor, a frequência respiratória média dos cavalos observada antes do exercício foi consistente com a frequência respiratória normal dos animais durante o repouso (Feitosa, 2004). Além disso, não foi encontrada correlação entre 0 Índice de Conforto Térmico e a frequência respiratória, demonstrando que os equinos não estavam sendo submetidos ao estresse por calor.

Yarnell (2011) analisou a temperatura ocular de cavalos a cada três horas durante o período de quatro dias consecutivos e não encontrou nenhuma relação entre a temperatura média ocular e a temperatura ambiente.

No entanto, a correlação linear positiva encontrada entre a frequência respiratória e a temperatura máxima ocular no presente estudo poderia possivelmente refletir a liberação de catecolaminas. Esses hormônios ajudam a preparar o animal para uma reação de "luta ou fuga", estão envolvidos no aumento da taxa respiratória e também promovem a redistribuição do fluxo sanguíneo durante situações estressantes. Williams et al. (2002) estudaram as respostas neuroendócrinas de equinos submetidos ao exercício em esteira durante condições climáticas favoráveis e em condições adversas (temperaturas elevadas e altos índices de humidade relativa), chegando a conclusão de que a adrenalina e a noradrenalina podem desempenhar um papel importante na adaptação de cavalos ao estresse térmico durante o treinamento.

\section{CONCLUSÃO}

Os resultados obtidos neste experimento são consistentes com os encontrados na literatura. Sendo assim, pode-se afirmar que a termografia infravermelha é uma ferramenta tecnológica versátil, não invasiva e de alta sensibilidade, capaz de medir alterações na temperatura máxima ocular em cavalos durante o treinamento de corrida. Entretanto, ainda são necessários mais estudos para determinar se esse parâmetro é capaz de refletir a intensidade da ativação dos sistemas alostáticos, o que poderia ser um bom indicador dos níveis de estresse. Para a determinação da natureza do estresse (euestresse ou distresse), é recomendável a utilização de outros parâmetros (análises comportamentais e parâmetros hematológicos) juntamente com o uso da câmera termográfica.

\section{NOTAS INFORMATIVAS}

Todos os procedimentos experimentais foram aprovados pelo Comitê de Ética para Experimentos em Animais da Universidade Federal do Paraná (UFPR, protocolo número 033/2017).

Os autores declaram não possuir conflito de interesses.

\section{REFERÊNCIAS}

BARTOLOMÉ, E; Sánchez, M.J.; Molina, A. et al. Using eye temperature and heart rate for stress assessment in young horses competing in jumping competitions and its possible influence on sport performance. Animal, v. 7, n. 12, p. 2044-2053, 2013. Disponível em: 
<https://doi.org/10.1017/S17517311130 01626> Acesso em: 18/03/2018. doi:10.1017/S1751731113001626

BLESSING, W.W. Lower brainstem pathways regulating sympathetically mediated changes in cutaneous blood flow. Cellular and Molecular Neurobiology, v. 23, n. 4, p. 527-538, $2003 . \quad$ Disponível em: https://doi.org/10.1023/A:102502002903 7> Acesso em: 18/03/2018. doi:10.1023/A:1025020029037

BRODY, S. Energetic efficiency of muscular work and indices of workreserve capacity. In Bioenergetics and Growth. Reinhold Publishing Corporation, New York, 1945. Cap. 24., p. 898-940.

CLARKE, A. F. A review of environmental and host factors in relation to equine respiratory disease. Equine Veterinary Journal, v. 19, n. 5, p. 435-441, 1987. Disponível em: https://doi.org/10.1111/j.2042-

3306.1987.tb02638.x> Acesso em: 18/03/2018.

CHURCH, J.S.; HEGADOREN, P.R.; PAETKAU, M.J. et al. Influence of environmental factors on infrared eye temperature measurements in cattle. Research in Veterinary Science, v. 96, n. 1, p. 220-226, 2014. Disponível em: https://www.sciencedirect.com/science/a rticle/pii/S0034528813003664> Acesso em: $\quad 18 / 03 / 2018 . \quad$ doi: 10.1016/j.rvsc.2013.11.006 2014

COOK, N.J.; SCHAEFER, A.L., WARREN, L. et al. Adrenocortical and metabolic responses to ACTH injection in horses: an assessment by salivary cortisol and infrared thermography of the eye. Canadian Journal of Animal Science, v. 81, p. 621, 2001. Disponível em:

$<$ https://www.researchgate.net/publicatio n/310749139_Adrenocortical_and_meta bolic_responses_to_ACTH_injection_in_ horses_an_assessment_by_salivary_cor tisol_and_infrared_thermography_of_the _eye> Acesso em: 18/03/2018.

HALL, C., BURTON, K.; MAYCOCK, E.; WRAGG, E. A preliminary study into the use of infrared thermography as a means of assessing the horse's response to different training methods. Journal of Veterinary Behavior: Clinical Applications and Research, v. 6, n. 5, p. 291-292, 2011. Disponível em: https://www.journalvetbehavior.com/artic le/S1558-7878(11)00075-X/abstract> Acesso em: 18/03/2018. doi: 10.1016/j.jveb.2011.05.005

McBRIDE, G.E.; CHRISTOPHERSON, R.J.; SAUER, W. Metabolic rate and plasma thyroid hormone concentrations of mature horses in response to changes in ambient temperature. Canadian Journal of Animal Science, v. 65 , n. 2 , p. 375-382, 1985. Disponível em: https://doi.org/10.4141/cjas85-043> Acesso em: 18/03/2018.

MCGREEVY, P.; WARREN-SMITH, A.; GUISARD, Y. The effect of double bridles and jaw-clamping crank nosebands on temperature of eyes and facial skin of horses. Journal of Veterinary Behavior: Clinical Applications and Research, v. 7, n. 3, p. 142-148, 2012. Disponível em: https://www.journalvetbehavior.com/artic le/S1558-7878(11)00143-

2/abstract?code=jveb-site $>$ Acesso em: 18/03/2018.

doi:

10.1016/j.jveb.2011.08.001

McKEEVER, K.H. The endocrine system and the challenge of exercise. Veterinary Clinics of North America: Equine Practice, v. 18, n. 2, p. 321-353, 2002. Disponível em: http://europepmc.org/abstract/med/1563 5911> Acesso em: Mar. 18,. doi: 10.1016/S0749-0739(02)00005-6

MOBERG, G.P. Biological response to stress: implications for animal welfare. 
In: The biology of animal stress. Wallingford: CAB International; 2000, Cap. 1, p. 1-21.

MORGAN, K.; EHRLEMARK, A.; SALLVIK, K. Dissipation of heat from standing horses exposed to ambient temperatures between $-3^{\circ} \mathrm{C}$ and $37^{\circ} \mathrm{C}$. Journal of Thermal Biology, v. 22, n. 3, p. 177-186, 1997.

PEETERS, M.; CLOSSON, C.; BECKERS, J. F. et al. Rider and horse salivary cortisol levels during competition and impact on performance. Journal of Equine Veterinary Science, v.33, p. 155-160, 2013. Disponível em: < https://www.sciencedirect.com/science/a rticle/pii/S0737080612002961> Acesso em: 15/07/2019.

PORGES, S.W. Cardiac vagal tone: a physiological index of stress. Neuroscience \& Biobehavioral Reviews, v. 19, n. 2, p. 225-233, 1995. Disponível

em: https://www.sciencedirect.com/science/a rticle/pii/014976349400066A> Acesso em: 18/03/2018. doi:10.1016/01497634(94)00066-A

SCHAEFER, A.L.; MATTHEWS, L.R.; COOL, N.J. et al. Novel non-invasive measures of animal welfare. In: Proceedings of Animal Welfare and Behavior: From Science to Solution; Joint NAWAC/ISAE Conference, Hamilton, New Zealand; 2002.

DE ASSIS SANTOS, F.; AZEVEDO, C.A.V. The Assistat Software Version 7.7 and its use in the analysis of experimental data. African Journal of Agricultural Research, v. 11, n. 39, p. 3733-3740, 2016. doi: 10.5897/AJAR2016.11522

STEWART, M.; WEBSTER, J.R.; SCHAEFER, A.L et al. Infrared thermography as a non-invasive tool to study animal welfare. Animal Welfare, v. 14, n. 4, p. 319-325, 2005. Disponível em:

<http://www.ingentaconnect.com/content /ufaw/aw/2005/00000014/00000004/art0 0007> Acesso em: 18/03/2018.

STEWART, M.; STAFFORD, K.J.; DOWLING, S.K. et al. Eye temperature and heart rate variability of calves disbudded with or without local anaesthetic. Physiology \& Behavior, v. 93, n. 4, p. 789-797, 2008. Disponível em:

https://www.sciencedirect.com/science/a rticle/abs/pii/S0031938407004787>

Acesso em: 18/03/2018. doi: 10.1016/j.physbeh.2007.11.044

VALERA, M.; BARTOLOMÉ, E.; SÁNCHEZ, M.J. et al. Changes in eye temperature and stress assessment in horses during show jumping competitions. Journal of Equine Veterinary Science, v. 32, n. 12, p. 827-830, 2012. Disponível em: https://www.j-evs.com/article/S07370806(12)00102-5/abstract> Acesso em: $18 / 03 / 2018$.

doi:

10.1016/j.jevs.2012.03.005

WILIAMS, R.J.; MARLIN, D.J.; SMITH, $\mathrm{N}$. et al. Effects of cool and hot humid environmental conditions on neuroendocrine responses of horses to treadmill exercise. The Veterinary Journal, v. 164, n. 1, p. 54-63, 2002. Disponível

em: https://www.sciencedirect.com/science/a rticle/pii/S1090023302907215> Acesso em: 18/03/2018. doi: 10.1053/tvjl.2002.0721

YARNELL, K. An investigation into the use of infrared thermography as a tool to assess the physiological stress response in the horse. Inglaterra, 2011. Dissertação (Doutorado em Filosofia) - Curso de pós-graduação em Fisiologia, Nottingham Trent University. Disponível em: http://irep.ntu.ac.uk/id/eprint/269/> Acesso em: 18/03/2018. 